\title{
DEVELOPMENT OF CRACK DETECTION METHOD WITH 2 DIMENSIONALLY GENERATED 3 DIMENSIONALLY RECONSTRUCTED IMAGES IN THT SOLDER JOINTS
}

\begin{abstract}
This work represents an interesting development in the detection and interpretation of crack evolution in through hole technology (THT) solder joints, which based on the development of general and common method. Serial sectioning is a useful method because it overcomes the problems associated with traditional two-dimensional metallographic techniques by providing information about (micro)structures in three-dimensions.

In our work, serials sectioning with reconstruction method was utilized to visualize the 3D nature of cracks in through hole solder joint. Accurate quantitive analysis of the cracks, such as crack length, position and extension are presented with a help of the developed method: newly defined parameter and serial-cross sectioning method.
\end{abstract}

Keywords: serial sectioning, reconstruction, total crack length (TCL), through hole technology (THT)

\section{Introduction}

Through Hole Technology (THT) is normally reserved for bulkier components such as electrolytic capacitors or semiconductors in large packages that require additional mounting strength to tolerate physical stress. Various reason for using through hole technology could be the size and shape of the component, the placement of leads, or the sensitivity and stress of the component. Through hole mounting provides stronger mechanical bonds than surface-mount technology techniques. However joint failure eventuates during operation as a result of various loadings. That means overloading causes fracture, long lasting permanent loading may induces creep, while cyclic loading generates fatigue. These causes may be distinguished, although the mechanisms often work simultaneously. In addition other causes, such as corrosion may play a role. In case of THT joint three crack locations (Fig. 1a) can be detected under fillet line (red line): cracks are close to pin (1), cracks in the bulk solder (2), cracks are close to hole (3). Solder joint failures are a result of crack initiation and growth. Failures of solders can be divided into two stages: the crack initiation stage, which starts with the first load cycle and ends when a detectable crack is present; and the crack propagation stage, which starts with a detectable crack and ends when the joint is open.

Some works [1-4] have shown that complete geometric and morphological information regarding a microstructure cannot be provided by two-dimensional metallographic analysis (3D eutectic grains). To provide as complete and unbiased description of a)

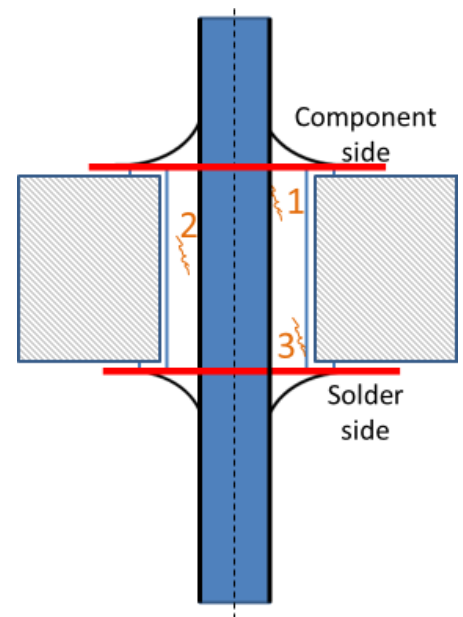

b)

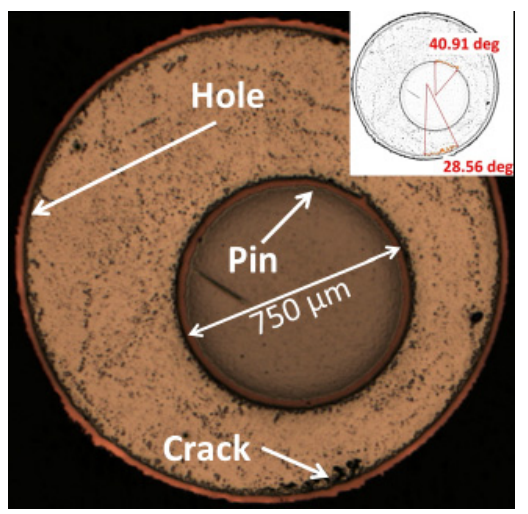

Fig. 1. a) Tipical crack locations in THT solder joints (longitudinal section), b) optical microscopy image of the serial cross-section and determination the dimension of cracks (angle)

\footnotetext{
INSTITUTE OF PHYSICAL METALLURGY, METALFORMING AND NANOTECHNOLOGY, MISKOLC, HUNGARY

** PALLASZ ATHÉNÉ UNIVERSITY, FACULTY OF GAMF TECHNICAL AND IT, KECSKEMÉT, HUNGARY

\# Corresponding author: femgreta@uni-miskolc.hu
} 
microstructure as possible, the field of materials characterization is gradually developing and adopting methods that provide quantitative microstructural information in three-dimensions (3D). One of them is serial sectioning [1]. This method is a widely used technique to recognize $3 \mathrm{D}$ characterization data at the macroand microscale. The procedure requires smooth surface, which can be accompolished by variety of methods: cutting, polishing, grinding, etching, etc. Constant depth of material removal has occurred between each section. To collect two-dimensional (2D) data/ image various technique can be applied. This method is typically realized using light microscopy [5], SEM [6,7] an electron probe microanalyzer [8] with serial sectioning method [9] and computer tomography $[1,7,10]$. However, as a metallographic tool, serial sectioning has some disadvantages. It is a time consuming and somewhat tedious procedure because it requires the repeated removal of layers of material, generally through grinding and polishing [1].

After collection of the series of 2D data files, computer software programs are used to construct 3D structure [11]. Reconstruct software developed by Department of Biology at Boston University is a free editor designed to facilitate montaging, alignment, analysis and visualization [1,12-14]. A large number of different structures can be easily traced and placed together in a single $3 \mathrm{D}$ scene that can be animated or saved [13].

According to available literature, very important to highlight, that serial section, reconstruction and CT methods and measurements [16-20] focus exclusively on visualization of voids - the size, the volume and distribution of this defect - and ignore cracks. However local stresses and strains and finally ultimate failures are also affected by cracks.

Use of longitudinal section is a general rule (common method) in industrial sector. However this common practice is not necessarily the best way to monitorize the crack propagation. Serial sectioning in radial direction (Serial cross-sectioning) provides better and more accurate examinations focusing the position (bulk or along the hole) and evolution of cracks. That is why we used serial cross- section technique combine with reconstruct software to characterize and visualize cracks in THT solder joint.

\section{Experimental procedure}

In this study through hole solder joints was characterized, serial cross-sectioned and reconstructed. Examined assemblies consisted of two (A and B sample) ELNA 1127 capacitors with 3+3 (A1, A2, A3 and B1, B2, B3) pins and a 6 layer PCB (Type: FR4). Diameter of pins were $750 \mu \mathrm{m}$. Solder material was SAC305. Joints were thermal cycle shock tested (Joints A: 4000 thermal cycles, joints B: 3000 thermal cycles). Joints were removed from PCB and embedded in epoxy resin. In order to carry out serial sectioning and achieve reproducible and controlled removal rate, some preliminary tests were carried out to determine the process parameter, e.g. time, load, speed.
P2000 SiC paper was used and the removal thickness of material was measured by micrometer. Table 1 shows the results of the determination process, i.e. set parameters.

TABLE 1

Set parameters used in serial cross-sectioning

\begin{tabular}{|c|c|c|c|c|}
\hline Section & $\begin{array}{l}\text { Removal } \\
\text { rate, } \mu \mathrm{m}\end{array}$ & Times, sec & Load, N & Speed, rpm \\
\hline 1 & \multirow{12}{*}{7} & 5 & \multirow{12}{*}{20} & \multirow{12}{*}{250} \\
\hline 2 & & 6 & & \\
\hline 3 & & 9 & & \\
\hline 4 & & 12 & & \\
\hline 5 & & 16 & & \\
\hline 6 & & 20 & & \\
\hline 7 & & 25 & & \\
\hline 8 & & 32 & & \\
\hline 9 & & 40 & & \\
\hline 10 & & 50 & & \\
\hline$\ldots$ & & & & \\
\hline 250 & & 50 & & \\
\hline
\end{tabular}

Serial (cross-) sectioning is a technique, which provide the gradual removal of parallel layers of material and the imaging of each layer. The procedure is described in following steps. Embedded joint was ground and polished using standard metallographic techniques. Thickness of the removal materials was set out a preliminary process, where the required time and load were determined to achieve $\approx 7 \mu \mathrm{m}$ material loss. During serial cross-sectioning $20 \mathrm{~N}$ force with various, enhanced process time were used. Optical microscope (Zeiss Axio Imager) image were taken from each section with mosaic technique. It was necessary because required magnification resulted small picture size/visual field and solder joint in whole can not be captured. With a help of mosaic technique bigger image size in higher magnification could be achieved. Fig. 1b shows a $8 \times 6$ mosaic serial image. Length of THT solder joint was $1750 \mu \mathrm{m}$, so 250 sections gave a depth of all volume of solder joint.

$3 \mathrm{D}$ reconstruction and imaging were performed by Reconstruct software, which was developed by the Department of Biology at Boston University. Reconstruct software can be freely available from SynapseWeb webpage [21]. Two offset points were made in the specimen to precisely align images. With a help of these points alignment by rotational and positional movements were occurred. Matched images were then imported to the Reconstruct software. This tool was used to edit and render the final three-dimensional images.

Three features were reconstructed from the THT solder joint: 1) hole, 2) pin and 3) cracks.

The internal observation was carried out by using an industrial computed tomograph GE Phoenix vtomex with $300 \mathrm{kV}$ microfocus tube and a 4 Megapixel flat panel detector dxr-250 with an active area of $400 \times 400 \mathrm{~mm}$, as well as a sub- $\mu$-computed tomography system GE Phoenix nanotom $\mathrm{m}{ }^{\circledR}$ with $180 \mathrm{kV}$ microfocus tube and flat panel detector for samples with $290 \mathrm{~mm}$ maximum diameter and $400 \mathrm{~mm}$ height. Cores were scanned 
using a full $360^{\circ}$ rotation of the sample. Digital radiographs were reconstructed using phoenix datos $x$ reconstruction software. Results of CT examination and $3 \mathrm{D}$ reconstruction were compared.

\section{Results and discussion}

Fig. 1b. shows an example of optical microscopy image of the serial sections from the THT solder joint. Same images were used ( $250 \mathrm{images} /$ joint) to produce 3D model of the joints. Fig. 3 presents a reconstructed 3D model from the 6 joints. Hole, pins and cracks were considered in the joint. The "empty space" in the model, which contains cracks and located between pin and hole is solder material (SAC 305). Both cracks (red and pink) were located near the pin. Red one have a $6^{*} 10^{-3} \mathrm{~mm}^{3}$ volume at solder side, while pink one $\left(\mathrm{V}=1 * 10^{-3} \mathrm{~mm}^{3}\right)$ is unfolded at component side.

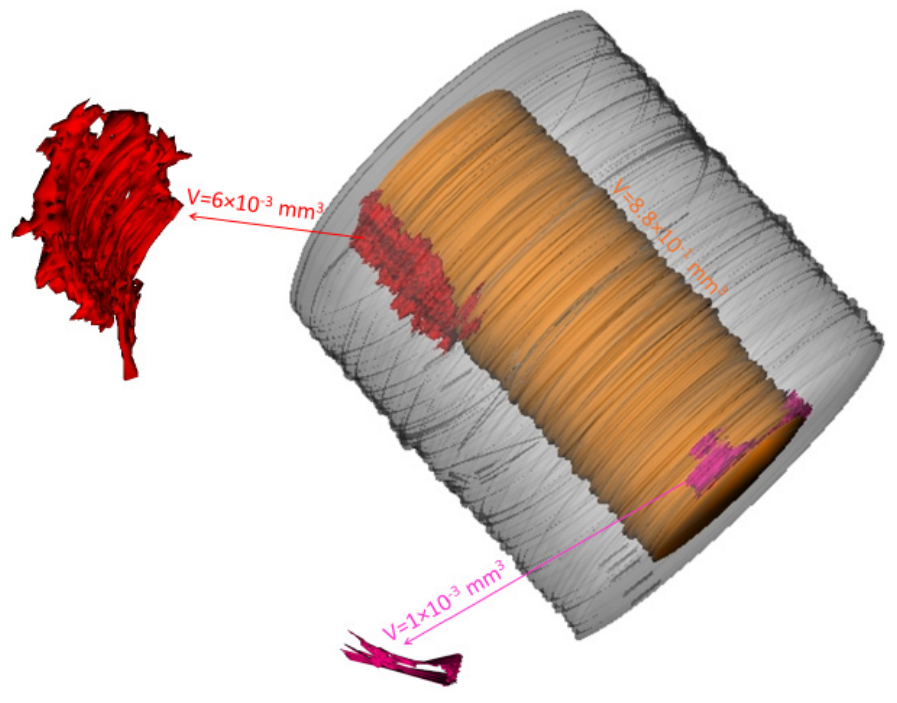

Fig. 2. Reconstructed 3D model of B2 joint

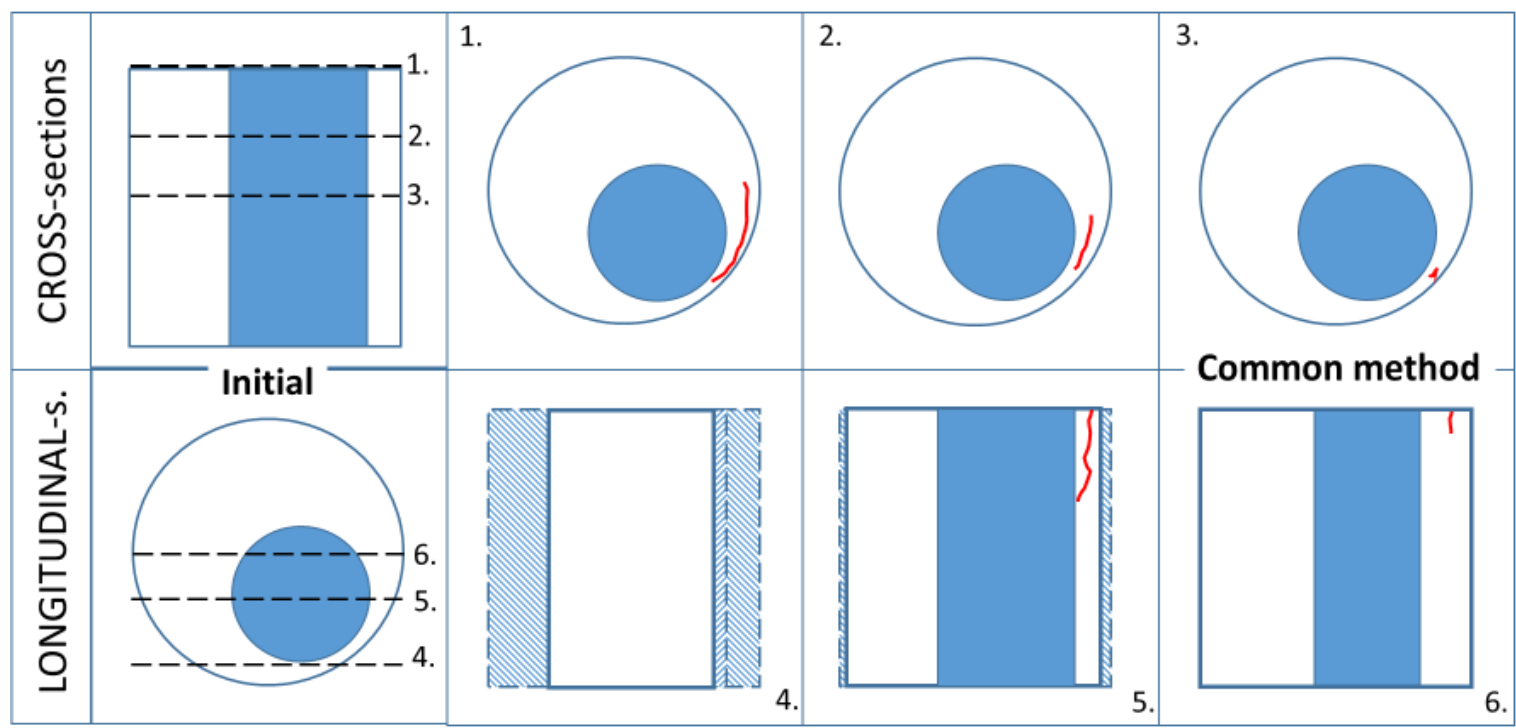

Fig. 3. Advantages of serial cross sectioning (picture 1-3) and difference between longitudinal sectioning (picture 4-6) and common method (picture 3,6) versus cross-sectioning method (picture 1-3)

Developed method is based on two pillars. One of the pillars is serial cross-sectioning combined reconstruct technique, which provide a full scale characterization of cracks in solder joints. Fig. 3. shows the advantages of serial cross-sectioning and the difference between longitudinal sectioning and common method versus cross sectioning. Fig. 3. visualizes the initial state, which was longitudinal and cross- sectioned. Picture 3 and 6 indicates the common method: one section in the middle planar of hole. This method provides only few details/data from the total crack(s). Pictures 4-6 represents longitudinal sections. Unfortunately significant parts/details of the cracks are lost, because the direction of the sections are not perpendicular to crack propagation, moreover parallel with them. In addition there is a real risk to lose the whole crack if it has a smaller width than the thickness of the removed material (distance between sections). In contrast, serial cross-sections (Picture 1-3), which set up 3D reconstruction images with other images (in our case
250 images), are settled down perpendicular to the direction of crack propagation, that is why this method provides accurate results with much less data/details loss.

In order to characterize the cracks (size, position, expansion), a new parameter was defined: Total Crack Length $=$ TCL. Necessary data were extracted from reconstruction. This parameter gives the second pillar of the developed method.

Calculation method for total crack length (TCL, \%) as follows (Eq.(1)):

$$
\begin{aligned}
& \max \left|\left(N_{L V}^{P S} \cdot D_{A V}\right) ;\left(N_{L V}{ }^{H S} \cdot D_{A V}\right)\right|+ \\
& T C L=\frac{+\max \left|\begin{array}{l}
\left.\left(N_{S}-N_{F V}{ }^{P C}\right) \cdot D_{A V}\right) ; \\
\left.\left(N_{S}-N_{F V}{ }^{H C}\right) \cdot D_{A V}\right)
\end{array}\right|}{T H_{P C B}} \cdot 100
\end{aligned}
$$


where: THPCB: thickness of the PCB, DAV: average distance between sections, NLV: last section number of visible crack, NFV: first section number of visible crack, NS: total number of sections, P: pin, H: hole, S: solder side, C: component side. Table 2 contains the TCL result of the joints.

The average distance between hole and pin shows the centralization of the pin. According to data on Fig. 4a, only one joint contained centralized pin (distance between hole and pin $=309 \mu \mathrm{m}$, hole diameter was $1500 \mu \mathrm{m}$ ), other joints were decentralized. Total crack lengths were varied in the range of
$62-95 \%$ in the 6 joints. Correlation coefficient $\left(R^{2}=0.75\right)$ shows a strong relationship between total crack length and centralization (distance between hole and pin). This means, the smaller is the distance between the hole and the pin, the greater is the length of the cracks. In other word: decentralization causes higher total crack length.

Total characterization of cracks involves the determination of maximal crack extension in the notable points of solder joint, i.e. sections in the planar of PCB at solder side and component side. All that were characterized by the angle between the middle

TCL and other measured parameters of joints

\begin{tabular}{|c|c|c|c|c|c|c|}
\hline \multirow{2}{*}{ Sample } & \multicolumn{2}{|c|}{ Solder side } & \multicolumn{2}{|c|}{ Component side } & \multirow{2}{*}{$\begin{array}{l}\text { Average distance } \\
\text { (Pin vs. Hole), } \mu \mathrm{m}\end{array}$} & \multirow{2}{*}{$\begin{array}{l}\text { Total crack length } \\
\text { (TCL), } \%\end{array}$} \\
\hline & Crack length, $\mu \mathrm{m}$ & Angle, rad & Crack length, $\mu \mathrm{m}$ & Angle, rad & & \\
\hline A1 & 1491 & 299 & 175 & 205 & 44 & 95,0 \\
\hline A2 & 833 & 262 & 560 & 223 & 105 & 79,6 \\
\hline $\mathbf{A 3}$ & 476 & 243 & 609 & 194 & 177 & 62,0 \\
\hline B1 & 1239 & 307 & 301 & 170 & 56 & 88,0 \\
\hline B2 & 637 & 197 & 490 & 182 & 309 & 64,0 \\
\hline B3 & 595 & 248 & 679 & 225 & 153 & 72,8 \\
\hline
\end{tabular}

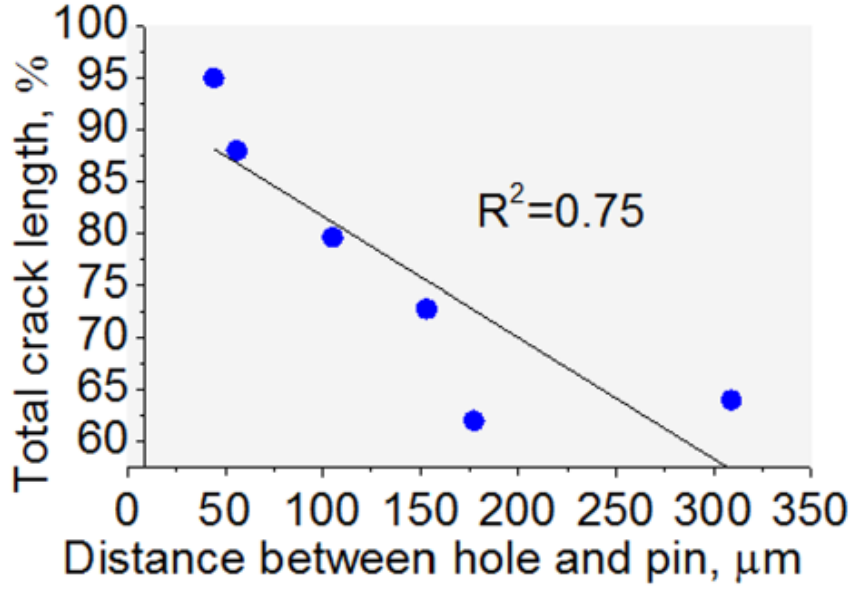

a)

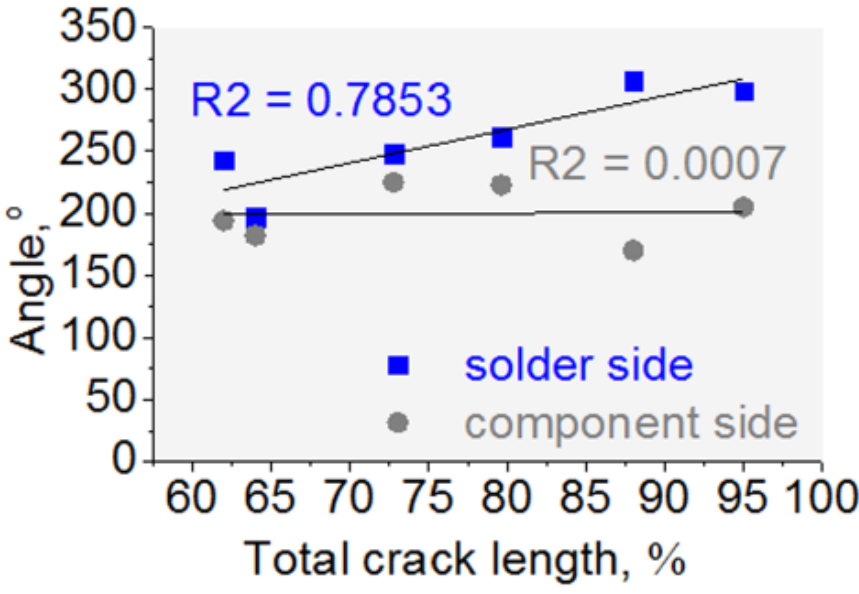

b)

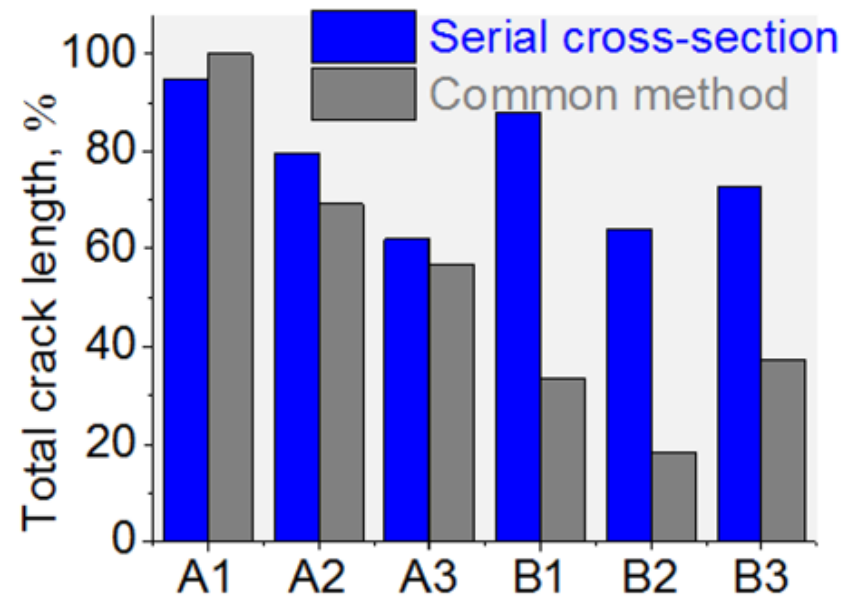

c)

Fig. 4. a) Total crack length versus centralization, b) Total crack length versus cracks dimensions, c) Comparison of total crack length measured with serial cross-section and common method (CT) 
axis of pin/ hole and width of the cracks (Fig. 1b). Average of the angles were determined in the selected sections and these values defined the dimension of cracks. It can be concluded, that expansion of cracks (angle) is higher in solder side than component side (Fig. 4b, Table 2). In case of solder side the dimension of cracks are strongly correlates to total crack length $\left(\mathrm{R}^{2}=0.7853\right)$. This correlation does not exist in the case of component side $\left(\mathrm{R}^{2}=0.0007\right)$. Based on this phenomena, with a help of further experiments a rating system can be constructed. Projected system would be a time (angle measurement in only one planar) and money saving method, because total crack length and lifetime would be predictable with a help of this small process (grinding and polishing in the planar of PCB and angle measurement).

The common method in industrial sector to determine total crack length can be summarized in below: grinding and polishing in the middle planar of THT joint and crack length measurement in this single planar. In this research, common method was realized by Computer Tomography (CT), i.e. the middle planar/ section of the joints were determined with a help of CT (Fig. 5). Fig. 4c shows smaller TCL in case of common method than in serial cross-section method. The reason for this can be the fact, that serial cross-section provides more data to determine total crack length, so uncertainty of this method is much smaller than common method. The difference is significant between the two method in case of joint B. Presumably the reason for this are other factors (heat expansion, voids, local microstructure) that affect the crack propagation.

\section{Conclusions}

Exploration and characterization of cracks formed in THT solder joints was carried out in this study. Developed method is based on two pillars: 1) the 3D structure of cracks was digitally reconstructed using image serial cross-sectioning and reconstruction software, 2) which was also used for quantitive analysis of the data $(\mathrm{TCL}=$ Total Crack Length) of cracks.

Serial-cross sectioning provides a full scale, accurate characterization of cracks and provides minimal data/ parts loss, while significant and remarkable data/parts are lost from crack with an application of longitudinal sectioning or common method.

A new parameter (Total Crack Length $=$ TCL) was defined, with a help of serial sectioning and reconstruction, in order to characterize the cracks (size, position, expansion). Following conclusions can be established:

1) The average distance between the hole and the pin shows the centralization of the pin. Strong relationship $\left(R^{2}=0.75\right)$ exist between TCL and centralization. This means, the smaller is the distance between the hole and the pin, the greater is the length of the cracks. In other word: decentralization causes higher total crack length.

2) TCL values were varied in the range of $62-95 \%$ in the examined joints.

3) Expansion of cracks (angle) is higher in solder side than component side. In case of solder side the dimension of cracks are strongly correlates to total crack length

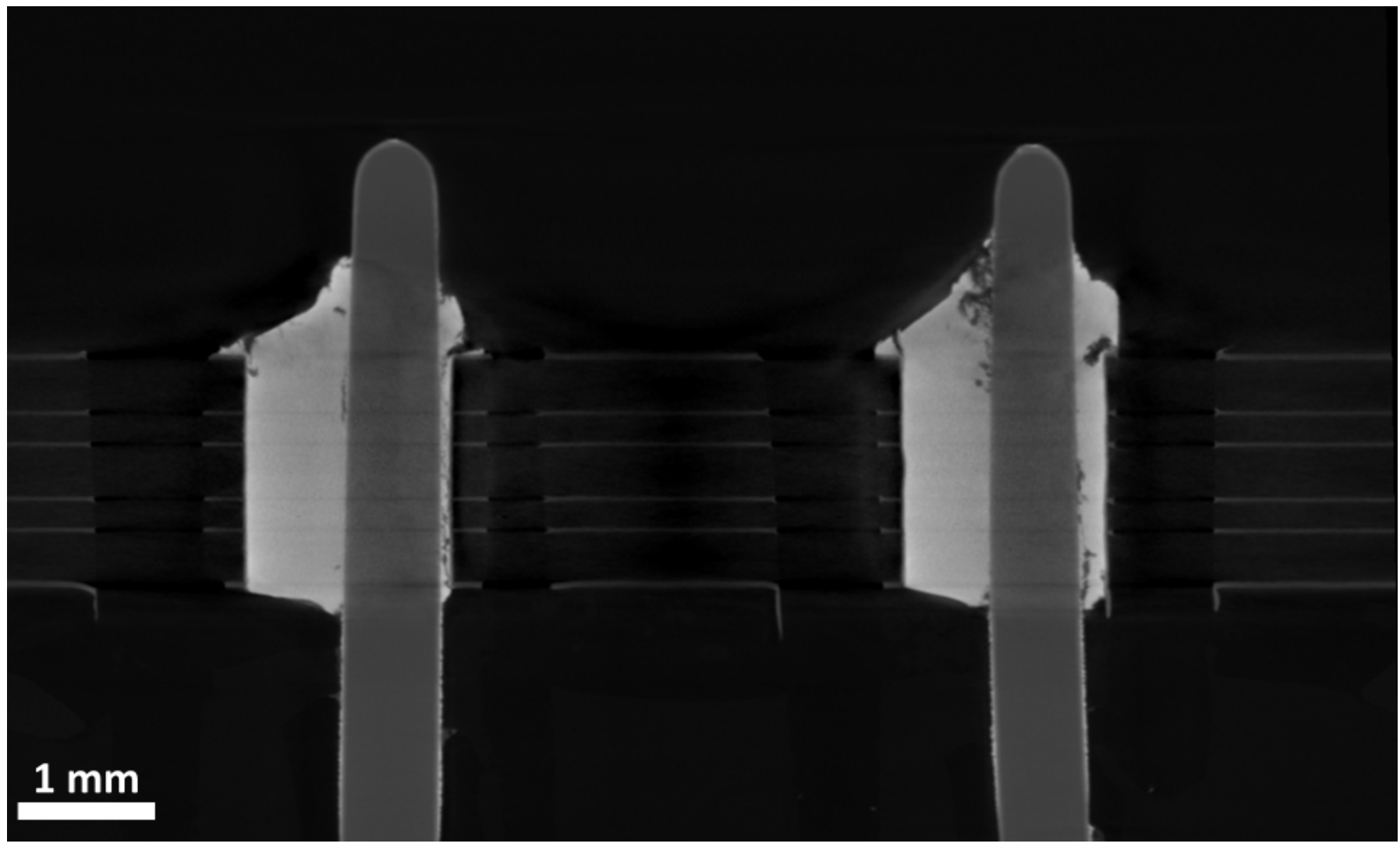

Fig. 5. CT image was used to determine middle planar of the joints (A1, A2) (common method) 
$\left(\mathrm{R}^{2}=0.7853\right)$. This correlation does not exist in the case of component side $\left(R^{2}=0.0007\right)$. Based on this phenomena, with a help of further experiments a rating system can be constructed. Projected system would be a time and money saving method, because TCL and lifetime would be predictable with a help of this small process (grinding and polishing in the planar of PCB and angle measurement).

4) TCL was smaller in case of common method than in serial cross-section method. The reason for this can be the fact, that serial cross-section provides more data to determine total crack length, so uncertainty of this method is much smaller than common method.

\section{Acknowledgments}

"The research work presented in this paper is based on the results achieved within the TÁMOP-4.2.1.B-10/2/KONV-2010-0001 project and carried out as part of the TÁMOP-4.2.2.A-11/1/KONV-2012-0019 project in the framework of the New Széchenyi Plan. The realization of this project was supported by the European Union, and co-financed by the European Social Fund. The authors would also like to show their gratitude to the TERPLÁN ZénóProgram supported by University of Miskolc."

\section{REFERENCES}

[1] C.M. Dinnis, A.K. Dahle, J.A. Taylor, Mat. Sci. and Eng. A 392, 440-448 (2005).

[2] M.V. Kral, G. Spanos, Acta Mater. 47, 711-724 (1999).
[3] B. Niroumand, K. Xia, Mater. Sci. Eng. A 283, 70-75 (2000).

[4] A. Tewari, A.M. Gokhale, Mater. Charact. 44, 259-269 (2000).

[5] R.H. Hopkins, R.W. Kraft, Trans AIME 233, 1526-1532 (1965).

[6] M.V. Krala, M.A. Mangan, G. Spanos, R.O. Rosenberg, Mat. Char. 45, 17-23 (2000).

[7] J. S. Thomsen, A. Laib , B. Koller, S. Prohaska, Li. Mosekilde, W. Gowin, J. of Microsc. 218, 171-179 (2005).

[8] R Marschallinger. Comp\& Geosci. 24, 875-883 (1998).

[9] F.M. L I a n g. World Hyphenation by Computer. PhD thesis, Stanford University, Stanford, CA 94305, June.

[10] O. Gauthier, R. Mullerb, D. Stechow, B. Lamy, P. Weiss, J.-M. Bouler, E. Aguado, G. Daculsi, Biomat. 26, 5444-5453 (2005).

[11] Michael D. Uchic. Comp. Meth. for Microst.-Prop. Relations. DOI 10.1007/978-1-4419-0643-4 2, Springer Science+Business Media, LLC 2011.

[12] J.C. Fiala, Convert, 1.11b, Boston University, 2001.

[13] J.C. Fiala, Sem Align, 1.26b, Boston University, 2001.

[14] J.C. Fiala, Igltrace, 1.26b, Boston University, 2001.

[15] J.C. Fiala, J. of Microsc. 218, 52-61 (2005).

[16] M.A. Dudek, L. Hunter, S. Kranz, J.J. Williams, S.H. Lau, N. Chawla, Mat. Charac. 61, 433-439 (2010).

[17] L. Jiang, N. Chawla, M. Pacheco, V. Noveski, Mat. Char. 62970-975 (2011).

[18] I. E. Anderson, J.L. Harringa, J. of Elect. Mat. 35, 1, 94-106 (2006).

[19] Luhua Xu, John H.L. Pang, Faxing Che, J. of Elect. Mat. 37, 6, 880-886 (2008)

[20] M. Yunus, K. Srihari, J.M. Pitarresi, A. Primaver, Micro. Reliability 43, 2077-2086 (2003).

[21] http://synapses.clm.utexas.edu/software-0 